\title{
Relation between reflux of bile acids into the stomach and gastric mucosal atrophy, intestinal metaplasia in biopsy specimens
}

\author{
Takeshi Matsuhisa* and Taku Tsukui \\ Department of Gastrointestinal Endoscopy, Tama-Nagayama University Hospital of Nippon Medical School, 1-7-1 Nagayama, Tama-city, Tokyo 206-8512, Japan
}

(Received 13 July, 2011; Accepted 30 July, 2011)

\begin{abstract}
During endoscopic examinations we collected fluid in the stomach that included reflux fluid from the duodenum, and assessed the effect of quantitatively determined bile acids on glandular atrophy and intestinal metaplasia using biopsy specimens. A total of 294 outpatients were enrolled in this study. Total bile acid concentration was measured by an enzyme immunoassay. Glandular atrophy and intestinal metaplasia scores were graded according to the Updated Sydney System. An effect of refluxed bile acids on atrophy and intestinal metaplasia was shown in the highconcentration reflux group in comparison with the control group. However, when the odds ratios (ORs) were calculated according to whether Helicobacter pylori $(H$. pylori) infection was present, no significant associations were shown between reflux bile acids and atrophy in either the $\boldsymbol{H}$. pylori-positive cases or -negative cases. The same was true for intestinal metaplasia in the $H$. pyloripositive cases, whereas intestinal metaplasia was more pronounced in the high-concentration reflux group in the $H$. pylorinegative cases (OR $2.4,95 \% \mathrm{Cl} 1.1-5.6)$. We could not clarify the effect of the reflux of bile acids into the stomach in the progression of atrophy. High-concentration bile acids had an effect on the progression of intestinal metaplasia in the $\boldsymbol{H}$. pylori-negative cases.
\end{abstract}

Key Words: atrophy, intestinal metaplasia, reflux bile acid

T $\mathrm{t}$ is known that the superficial gastritis that occurs in association with Helicobacter pylori $(H$. pylori) infection progresses to atrophic gastritis, and that over time intestinal metaplasia occurs, ${ }^{(1)}$ and stomach cancer develops. Excessive salt intake, insufficient intake of fresh fruit and vegetables, etc., as well as $H$. pylori has been demonstrated to play an important role as factors in the progression of atrophic gastritis, ${ }^{(2)}$ and there is also a report in relation to a synergistic effect of refluxed bile acids and $H$. pylori. ${ }^{(3)}$ On the other hand, the incidence of the cancer in the remnant stomach after Billroth II reconstruction is higher than after Billroth I reconstruction. ${ }^{(4)}$ An effect of reflux of bilecontaining duodenal fluid has been pointed out as the incidence of the cancer, ${ }^{(5)}$ and it has been confirmed in animal experiments. ${ }^{(6)}$

However, there have been few studies on the effect of bile acids that reflux into the stomach in association with duodenogastric reflux (DGR) on atrophy and intestinal metaplasia in cases with no history of gastric resection. ${ }^{(7,8)} \mathrm{We}$ therefore collected intragastric juice containing refluxed bile acids from the duodenum in subjects undergoing an endoscopic examination and investigated whether there was an association between refluxed bile acids and either atrophy or intestinal metaplasia in biopsy tissue. We also observed the relationship between $H$. pylori infection and atrophic gastritis based on serum pepsinogen values.

\section{Materials and Methods}

Materials. A total of 294 outpatients (mean age 58.7 years, range $20-86$ years, sex ratio 182:112) who were examined at the Tama-Nagayama University Hospital of Nippon Medical School were enrolled in this study. The survey of the gastric mucosa that included collection of gastric juice and diagnosis of $H$. pylori infection was being an adult 20 years of age or over. An upper gastroduodenal endoscopic examination was performed in every subject. Subjects who had a history of gastric surgery or pregnancy, who fulfilled any of the exclusion criteria below, or who were judged to be unsuitable because it might be impossible to perform an endoscopic examination were excluded from this study. This study was approved by the ethics committee of TamaNagayama University Hospital of Nippon Medical School, and written informed consent was obtained from all subjects.

Gastric juice collection. The endoscopic examinations were performed in the early morning after an overnight fast, without ingesting any food or liquids or taking any medication. When the examinations were performed, no gastric mucolytic and mucus removing agents, foaming mucus removing agents, or gastrointestinal motility inhibitors were used, and the subjects were asked to expectorate all of the local anesthetic that was used for pharyngeal anesthesia. In order to prevent postural reflux of fluid in the duodenum into the stomach, the subjects were maintained in the seated position until just before the start of the examination. Whenever reflux of duodenal fluid into the stomach through the pyloric ring was detected during the examination of the stomach, the subject was excluded as a subject in order to eliminate effects associated with posture during the examination, i.e., the left lateral position.

As much of the gastric contents that had collected in the fornix or greater curvature side of the body of the stomach as possible was aspirated immediately before the endoscopic examination. The gastric juice was aspirated via the forceps channel in the endoscope and captured in a recovery vessel. Whenever a bleeding lesion was observed or whenever the mucosa was injured during aspiration of the gastric juice and bleeding was observed, the subject was excluded as a subject because of the possibility of measuring bile acids in the blood that had commingled with the gastric juice. After the collection of gastric contents, gastric mucolytic and mucus removing agents, foaming mucus removing agents were used.

Measurement of the bile acid concentration. The fluid that had been collected was immediately frozen and stored $\left(-20^{\circ} \mathrm{C}\right)$, and the total bile acid concentration was measured by an enzy-

*To whom correspondence should be addressed.

E-mail: matuhisa@nms.ac.jp 
matic assay (Daiichi Pure Chem. Co., Ltd., Tokyo, Japan) ${ }^{(9)}$ by the outsource (SRL, Inc., Tachikawa, Japan).

Serum pepsinogen values. Pepsinogen (PG) I and II values were measured in serum collected before the endoscopic examination, and the I/II ratio was calculated. The PG I and II measurements were made by means of a chemiluminescent enzyme immunoassay (CLEIA). When the PG I value was $\leq 70 \mathrm{ng} / \mathrm{ml}$ and the I/II ratio was $\leq 3.0$, the specimen was recorded as PG-positive, otherwise it was recorded as PG-negative. ${ }^{(10)}$

Diagnosis of atrophy, intestinal metaplasia, and $\boldsymbol{H}$. pylori infection. Biopsy specimens for histological diagnosis, including of $H$. pylori infection, were collected from the greater curvature of the distal antrum (\#1), the greater curvature of the proximal body (\#2), and the lesser curvature of the distal body (\#3) in accordance with the triple-site gastric biopsy method. ${ }^{(11,12)}$ Hematoxylin-eosin staining was performed to make the histological diagnosis. Giemsa stain and $H$. pylori-specific antibody immune stain (Dako, Clostrup, Denmark) in selected cases were used to diagnose $H$. pylori infection.

Glandular atrophy, intestinal metaplasia, and $H$. pylori scores were graded according to the Updated Sydney System into the following four grades as follows: 0, none; 1, mild; 2, moderate; and 3 , severe. ${ }^{(3)}$ When the grade of atrophy, intestinal metaplasia, or $H$. pylori infection was 0 , the subject was classified as negative that parameter, and when the score for atrophy, intestinal metaplasia, or $H$. pylori infection was 1,2 , or 3 , the subject was classified as positive for that parameter. The histological diagnosis in the biopsy specimens was made by one pathologist (Yamada N.) using the same criteria.

Statistical analysis. The mean bile acid concentrations were expressed as mean \pm standard error (SE).

The subjects whose total bile acid concentration was below the sensitivity of detection by the assay $(3 \mathrm{nmol} / \mathrm{ml}$ ) were assigned to the control group, and the subjects whose concentrations were above the sensitivity of detection were divided into 3 equal groups starting with the subjects who had the lowest concentrations: Group A (0-33\%), Group B (34-66\%), and Group C (67-100\%).
The odds ratios (ORs) and 95\% confidence intervals (CIs) of groups $\mathrm{A}, \mathrm{B}$, and $\mathrm{C}$, when the odds for the occurrence of atrophy, occurrence of intestinal metaplasia, and $H$. pylori infection in the control group were set equal to 1 , were each calculated. Similarly, the OR and $95 \% \mathrm{CI}$ of the H. pylori-positive cases when the PGpositive odds of the $H$-pylori-negative cases was set equal to 1 , and the intestinal metaplasia-positive OR and 95\%CI when the occurrence of atrophy odds of the intestinal-metaplasia-negative cases was set equal to 1 , were calculated. The $95 \%$ CIs of the ORs were calculated by the Woolf's method. ${ }^{(13)}$

\section{Results}

The 294 subjects consisted of 6 patients with early stomach cancer, 2 patients with gastric mucosa-associated lymphoid tissue (MALT) lymphoma, 104 patients with peptic ulcer disease, 21 patients gastric polyp, 78 patients gastritis (erosive gastritis, gastric erosion, superficial gastritis, and hemorrhagic gastritis), 4 patients with reflux esophagitis, and 79 normal group subjects who had no evidence of any local lesions (including atrophic gastritis) in the upper gastrointestinal tract (Table 1).

Bile acid reflux was observed in $189(64.3 \%)$ of the 294 subjects of the study. No significant differences in incidence of bile acid reflux or in bile acid concentration were found among the 3 groups containing the largest numbers of subjects: the peptic ulcer disease group, the normal group, and the gastritis group (Table 1).

The rate of $H$. pylori infection among the subjects was $27.2 \%$. No associations were found between the presence or absence of $H$. pylori infection and the refluxed bile acid concentration in the comparison between the control group and any of Group A, Group B, or Group C (Table 2).

PG-positive subjects were more common among the $H$. pyloripositive subjects than among the $H$. pylori-negative subjects (OR 7.9, 95\%CI 4.3-14.7) (Table 3).

According to the pathological diagnosis by the triple-site biopsy method, there was a stronger tendency for intestinal metaplasia to be seen among the subjects whose biopsies showed atrophy in

Table 1. Endoscopic diagnosis, concentration of bile acids in gastric juice, incidence of bile acid reflux into the stomach

\begin{tabular}{|c|c|c|c|}
\hline Endoscopic diagnosis & $n$ & Bile acid concentration (mean \pm SD) & Incidence of reflux \\
\hline Gastric cancer & 6 & & \\
\hline Gastric MALToma & 2 & & \\
\hline Peptic ulcer disease & 104 & $186.8 \pm 320.3 \mathrm{nmol} / \mathrm{ml}^{*}$ & $73.1 \% * * * *$ \\
\hline Gastric polyp & 21 & & \\
\hline Gastritis & 78 & $347.0 \pm 1072.4 \mathrm{nmol} / \mathrm{ml}^{* *}$ & $60.3 \% * * * * *$ \\
\hline Reflux esophagitis & 4 & & \\
\hline Normal group & 79 & $323.7 \pm 989.5 \mathrm{nmol} / \mathrm{ml}$ *** & $64.6 \% * * * * * *$ \\
\hline Total & 294 & $250.0 \pm 780.7 \mathrm{nmol} / \mathrm{ml}$ & $64.3 \%$ \\
\hline
\end{tabular}

Table 2. Relation between $H$. pylori infection and the refluxed bile acid cocentration

\begin{tabular}{ccccccc}
\hline \multirow{2}{*}{ Group } & \multirow{2}{*}{$n$} & \multicolumn{2}{c}{ H. pylori infection } & \multirow{2}{*}{ Odds } & OR & \multirow{2}{*}{$95 \% \mathrm{Cl}$} \\
\cline { 3 - 4 } & & Negative & Positive & & & \\
\hline Control & 105 & 74 & 31 & 0.419 & 1.0 & \\
A & 61 & 46 & 15 & 0.326 & 0.8 & $0.4-1.6$ \\
B & 65 & 49 & 16 & 0.327 & 0.8 & $0.4-1.6$ \\
C & 63 & 45 & 18 & 0.400 & 1.0 & $0.5-1.9$ \\
\hline
\end{tabular}

No associations were found between the presence or absence of $H$. pylori infection and the refluxed bile acid concentration in the comparison between the control group and any of Group A, Group B, or Group C. 
specimens \#1 and \#3 than among the subjects whose biopsies did not (Table 4).

Based on the results of the total bile acid concentrations measurements, there were 105 subjects in the control group, 61 in Group A (3-29 nmol/ml), 65 in Group B (30-199 nmol/ml), and 63 in Group C (200 nmol/ml and over).

Atrophy and intestinal metaplasia in specimen \#1 were marked in Group $\mathrm{C}$ in comparison with the control group (atrophy: OR 5.1, 95\%CI 1.2-21.7; intestinal metaplasia: OR 2.3, 95\%CI 1.14.6), but no significant differences were found between either
Group A or Group B and the control group. When the results were examined according to whether $H$. pylori infection was present, no significant differences in atrophy from the control group were found in either the $H$. pylori-positive group or the $H$. pylorinegative group (Table 5). The same was true in regard to intestinal metaplasia in the $H$. pylori-positive group (Table 5). In the $H$. pylori-negative group, however, intestinal metaplasia was more marked in Group $\mathrm{C}$ than in the control group (OR 2.4, 95\%CI 1.1-5.6) (Table 5).

Table 3. Relation between the results of the pepsinogen (PG) test and $H$. pylori infection

\begin{tabular}{|c|c|c|c|c|c|c|}
\hline \multirow{2}{*}{ H. pylori infection } & \multirow{2}{*}{$n$} & \multicolumn{2}{|c|}{ PG test } & \multirow{2}{*}{ Odds } & \multirow{2}{*}{ OR } & \multirow{2}{*}{$95 \% \mathrm{Cl}$} \\
\hline & & Negative & Positive & & & \\
\hline Negative & 214 & 192 & 22 & 0.115 & 1.0 & \\
\hline Positive & 80 & 42 & 38 & 0.905 & 7.9 & $4.3-14.7$ \\
\hline
\end{tabular}

PG-positive subjects were more common among the $H$. pylori-positive subjects than among the $H$. pylori-negative subjects.

Table 4. Relation between glandular atrophy and intestinal metaplasia according to the results of histological examination of biopsy specimens obtained by the triple-site biopsy method

\begin{tabular}{|c|c|c|c|c|c|c|c|c|}
\hline \multirow{2}{*}{\multicolumn{2}{|c|}{ Specimen number }} & \multirow{2}{*}{$\begin{array}{l}\text { Intestinal } \\
\text { metaplasia }\end{array}$} & \multirow{2}{*}{$n$} & \multicolumn{2}{|c|}{ Glandular atrophy } & \multirow{2}{*}{ Odds } & \multirow{2}{*}{ OR } & \multirow{2}{*}{$95 \% \mathrm{Cl}$} \\
\hline & & & & Negative & Positive & & & \\
\hline \multirow{2}{*}{$\# 1$} & \multirow{2}{*}{ greater curvature of the distal antrum } & Negative & 115 & 109 & 6 & 0.055 & 1.0 & \\
\hline & & Positive & 18 & 5 & 13 & 2.600 & 47.3 & $12.6-176.6$ \\
\hline \multirow{2}{*}{$\# 2$} & \multirow{2}{*}{ greater curvature of the proximal body } & Negative & 186 & 178 & 8 & 0.045 & 1.0 & \\
\hline & & Positive & 1 & 1 & 0 & & & \\
\hline \multirow{2}{*}{ \#3 } & \multirow{2}{*}{ lesser curvature of the distal body } & Negative & 113 & 96 & 17 & 0.177 & 1.0 & \\
\hline & & Positive & 48 & 5 & 43 & 8.600 & 48.6 & $16.8-140.2$ \\
\hline
\end{tabular}

According to the pathological diagnosis by the triple-site biopsy method, there was a stronger tendency for intestinal metaplasia to be seen among the subjects whose biopsies showed atrophy in specimens \#1 and \#3 than among the subjects whose biopsies did not.

Table 5. Glandular atrophy (A) and intestinal metaplasia (B) according to the bile acid concentration and according to whether $H$. pylori infection was present or absent

\begin{tabular}{|c|c|c|c|c|c|c|c|}
\hline \multirow{2}{*}{ (A) } & \multirow{2}{*}{ Group } & \multirow{2}{*}{$n$} & \multicolumn{2}{|c|}{ Glandular atrophy } & \multirow{2}{*}{ Odds } & \multirow{2}{*}{ OR } & \multirow{2}{*}{$95 \% \mathrm{Cl}$} \\
\hline & & & Negative & Positive & & & \\
\hline \multirow{4}{*}{ Total } & Control & 105 & 46 & 3 & 0.065 & 1.0 & \\
\hline & $A$ & 61 & 26 & 3 & 0.115 & 1.8 & $0.3-9.4$ \\
\hline & B & 65 & 21 & 6 & 0.286 & 4.4 & $0.99-19.2$ \\
\hline & $\mathrm{C}$ & 63 & 21 & 7 & 0.333 & 5.1 & $1.2-21.7$ \\
\hline \multirow{2}{*}{ H. pylori (+) } & Control & 26 & 8 & 1 & 0.125 & 1.0 & \\
\hline & C & 19 & 4 & 5 & 1.250 & 10.0 & $0.9-117.0$ \\
\hline \multirow{2}{*}{ H. pylori (-) } & Control & 79 & 38 & 2 & 0.053 & 1.0 & \\
\hline & $\mathrm{C}$ & 44 & 17 & 2 & 0.118 & 2.2 & $0.3-17.2$ \\
\hline \multirow{2}{*}{ (B) } & \multirow{2}{*}{ Group } & \multirow{2}{*}{$n$} & \multicolumn{2}{|c|}{ Intestinal metaplasia } & Odds & $\mathrm{OR}$ & $95 \% \Omega$ \\
\hline & & & Negative & Positive & Odds & OR & $95 \% \mathrm{Cl}$ \\
\hline \multirow{4}{*}{ Total } & Control & 105 & 84 & 21 & 0.250 & 1.0 & \\
\hline & $A$ & 61 & 52 & 9 & 0.173 & 0.7 & $0.3-1.6$ \\
\hline & B & 65 & 49 & 16 & 0.327 & 1.3 & $0.6-2.7$ \\
\hline & $\mathrm{C}$ & 63 & 40 & 23 & 0.575 & 2.3 & $1.1-4.6$ \\
\hline \multirow{2}{*}{ H. pylori (+) } & Control & 26 & 20 & 6 & 0.300 & 1.0 & \\
\hline & $C$ & 19 & 12 & 7 & 0.583 & 1.9 & $0.5-7.2$ \\
\hline \multirow{2}{*}{ H. pylori (-) } & Control & 79 & 64 & 15 & 0.234 & 1.0 & \\
\hline & C & 44 & 28 & 16 & 0.571 & 2.4 & $1.1-5.6$ \\
\hline
\end{tabular}

Atrophy and intestinal metaplasia in specimen \#1 were marked in Group C in comparison with the control group, but no significant differences were found between either Group A or Group B and the control group. When the results were examined according to whether $H$. pylori infection was present, no significant differences in atrophy from the control group were found in either the $H$. pylori-positive group or the $H$. pylori-negative group. The same was true in regard to intestinal metaplasia in the $H$. pylori-positive group. In the $H$. pylori-negative group, however, intestinal metaplasia was more marked in Group C than in the control group. 


\section{Discussion}

The main cause of chronic atrophic gastritis, which is prevalent among Japanese, has been found to be related to $H$. pylori. ${ }^{(14)}$ Intestinal metaplasia, which is characterized by the occurrence of goblet cells, absorptive epithelial cells, and, at times, Paneth cells is another change that is frequently seen in the gastric mucosa of Japanese. ${ }^{(3)}$ Intestinal metaplasia is known to be a change that occurs secondary to chronic atrophic gastritis, ${ }^{(15)}$ but the factors that contribute to intestinal metaplasia and its significance are unclear. In 1987 the authors reported an association between refluxed bile acids in the stomach and the endoscopic form of the pylorus. ${ }^{(16)}$ In the present study we investigated whether refluxed bile acids in the stomach affect the progression of atrophy and intestinal metaplasia.

Old studies found higher refluxed bile acid concentrations in gastric ulcer groups than in control groups, ${ }^{(17,18)}$ and there is a report that the higher concentrations are due to a decrease in gastric secretion not due to increased DGR. ${ }^{(19)}$ On the other hand, no differences were found in secondary bile acid composition, including deoxycholic acid, between stomach cancer patients and a control group. ${ }^{(20)}$ Even in our own study, no differences in the incidence of reflux or in its concentration were seen among the peptic ulcer disease group, gastritis group, and normal group. We therefore carried out our study of reflux bile acids regardless of the disease. That was because atrophic gastritis and intestinal metaplasia are often seen in Japanese, and the purpose of our study was to elucidate the relation between the gastric mucosa and bile acids.

The gastric juice collection during the endoscopic examination was performed in the morning after an overnight fast. However, since the examination time varied with the subject and there were differences in the degree of the patients' anxiety the examination, it is almost impossible to a condition the same between subjects. Moreover, there are investigators who question the reproducibility of the refluxed bile acids, ${ }^{(7)}$ and because the present study was a cross-sectional study, we did not take reproducibility into account.

Bile acids have been experimentally confirmed to inhibit the growth of $H$. pylori, ${ }^{(21)}$ and $H$. pylori are known to be eradicated in patients who have undergone gastric surgery. ${ }^{(22)}$ In our own study, which was conducted on subjects who had not undergone gastric resection, no decrease in $H$. pylori infection rate was shown even in Group $\mathrm{C}$, which had a high reflux bile acid concentration. A study of patients with dyspepsia or gastritis reported no significant difference in $H$. pylori infection rate between a group with bile acid reflux and a group without bile acid reflux (36.9\% and 54.5\%, respectively), ${ }^{(23)}$ and thus the results were similar to our own. In contrast to the postoperative stomach, the refluxed bile acids are drained into the duodenum by pyloric function in unresected patients. We think they have no effect in decreasing the $H$. pylori infection rate.

It is an unquestioned fact that long-term $H$. pylori infection greatly contributes to the progression and expansion of atrophic gastritis. ${ }^{(24)}$ The PG method measures the serum PG concentration, and is used to pick up persons with atrophic gastritis, which is a high-risk group for stomach cancer. It is widely used for gastric cancer screening in Japan as objective and correct serodiagnostic method for atrophic gastritis. ${ }^{(25)}$ The results of our study also showed significantly more PG-positive subjects in the $H$. pyloripositive group (OR: 7.9, 95\%CI 4.3-14.7), thereby corroborating the results indicating that $H$. pylori infection has an effect on atrophy.

A review of the relationship between atrophic gastritis and intestinal metaplasia in the greater curvature of the distal antrum (biopsy specimen \#1) and lesser curvature of the distal body (biopsy specimen \#3) showed that the atrophy-positive odds were higher in the intestinal metaplasia-positive group than in the intestinal metaplasia-negative group. Atrophic gastritis spreads from the pylorus to the body of the stomach with increasing age, ${ }^{(26)}$ and intestinal metaplasia develops over the course of 10 or so years after the atrophic changes appear. ${ }^{(27)}$ Intestinal metaplasia starts in the pyloric gland region, and it mainly ascends the lesser curvature gradually and in a linear fashion to the fundic gland mucosa, and in the fundic gland mucosa it spreads from the lesser curvature to the greater curvature. ${ }^{(28)}$ It is possible to understand the results in biopsy specimens \#1 and \#3 in our study on the basis of these facts as well.

Based on the fact that many differentiated stomach cancers develop in gastric mucosa that exhibit intestinal metaplasia, Matsukura et al. ${ }^{(20)}$ reported that intestinal metaplasia in the gastric mucosa is a precancerous condition. In a study by Uemura et al. ${ }^{(30)}$ there was a high rate of development of stomach cancer in subjects with $H$. pylori infection, especially in patients with severe atrophic gastritis and severe intestinal metaplasia. On the other hand, although most differentiated stomach cancers are associated with intestinal metaplasia around them, there are objections to the view that regards intestinal metaplasia as a precancerous condition. ${ }^{(31)}$ Tatematsu et al. ${ }^{(32)}$ have stated that the intestinal metaplasia that is seen in the process of progression of atrophic gastritis is an abnormality of differentiation that occurs at the stem cell level, that $H$. pylori infection promotes this process, that cancer is a gene abnormality that occurs in precursor cells that have differentiated from stem cell, and that intestinal metaplasia and carcinogenesis are separate phenomena that occur in parallel. Moreover, because $H$. pylori infection is not observed in mucosa affected by intestinal metaplasia, there is also the view that intestinal metaplasia in the gastric mucosa is an aggressive immunological adaptation phenomenon by the body to $H$. pylori infection. ${ }^{(33)}$

As the antrum is readily affected by DGR, atrophy and intestinal metaplasia is often recognized with endoscopic examination and histological diagnosis. For this reason, we used specimen \#1 (the distal antrum) to investigate the effect of bile acids on atrophy and intestinal metaplasia. It has been shown that bile acids may affect atrophy of the gastric mucosa, but when examined according to whether $H$. pylori infection was present or not, no effect of bile acids was observed in either a positive group or a negative group. It was impossible to demonstrate synergism between bile acids and $H$. pylori ${ }^{(3)}$ in the $H$. pylori-positive subjects by our results. Because of the powerful effect of $H$. pylori infection as a risk factor for atrophy, the possibility that the effect of bile acids is concealed cannot be ruled out. An effect of bile acids on intestinal metaplasia has also been shown, but when examined according to whether $H$. pylori was present, no effect of bile acids on intestinal metaplasia was seen in the $H$. pylori-positive group. Nakamura et al. have stated the possibility of the progression of intestinal metaplasia is affected by DGR. ${ }^{(8)}$ The fact that there were few H. pylori-positive subjects in 294 subjects appears to be one of the factors involved in why no association between reflux bile acids and intestinal metaplasia was found in the $H$. pylori-positive group. The H. pylori infection rate among persons who underwent an upper gastrointestinal endoscopic examination in our department was approximately 53\% (data not shown). Many young subjects, who have a low rate of $H$. pylori infection, were included in this study among the subjects who received a stomach screening examination at another institution and received a second endoscopic examination based on the results. On the other hand, a high concentration of bile acids was found to affect intestinal metaplasia in the H. pylori-negative group. Tatematsu et al. have stated that intestinal metaplasia is promoted by H. pylori, ${ }^{(32)}$ but it is also induced by antigen stimuli other than H. pylori. ${ }^{(33)}$ Our results that intestinal metaplasia occurs in the high bile acid concentration group of $H$. pylori-negative subjects support this. An effect of reflux bile acids on intestinal metaplasia and carcinogenesis has been shown in animal experiments. ${ }^{\text {(34) }}$ Another report states pancreaticoduodenal secretions is implicated in gastric carcinogenesis in the rat. ${ }^{(35)}$ A study that includes bile 
acids and pancreaticoduodenal secretions seems to be needed.

In conclusion, $H$. pylori infection plays a major role in the progression of atrophy, but it was impossible to demonstrate an effect of bile acid reflux into the stomach. High concentrations of bile acids were shown to have an effect on the progression of intestinal metaplasia in $H$. pylori-negative subjects, but there was no clear effect of bile acids in positive subjects. Based on our experience in this study, we plan to conduct a study on a large number of subjects in a multi-center cooperative study in Japan.

\section{References}

1 Kekki M, Siurala M, Varis K, Sipponen P, Sistonen P, Nevanlinna HR. Classification principles and genetic of chronic gastritis. Scand J Gastroenterol Suppl 1987; 141: 1-28.

2 Correa P. Chronic gastritis: a clinico-pathological classification. Am J Gastroenterol 1988; 83: 504-509.

3 Dixon MF, Genta RM, Yardley JH, Correa P. Classification and grading of gastritis. The Updated Sydney System. International Workshop on the Histopathology of Gastritis, Houston 1994. Am J Surg Pathol 1996; 20: 1161-1181.

4 Northfield TC, Hall CN. Carcinoma of the gastric stump: risks and pathogenesis. Gut 1990; 31: 1217-1219.

5 Orlando R 3rd, Welch JP. Carcinoma of the stomach after gastric operation. Am J Surg 1981; 141: 487-491.

6 Kondo K, Suzuki H, Nagayo T. The influence of gastro-jejunal anastomosis on gastric carcinogenesis in rats. Gann 1984; 75: 362-369.

7 Sobala GM, O'Connor HJ, Dewar EP, King RFG, Axon AT, Dixon MF. Bile reflux and intestinal metaplasia in gastric mucosa. J Clin Pathol 1993; 46: 235-240.

8 Nakamura M, Haruma K, Kamada T, et al. Duodenogastric reflux is associated with antral metaplasic gasiritis. Gastrointest Endosc 2001; 53: 53-59.

9 Mashige F, Tanaka N, Maki A, Kamei S, Yamanaka M. Direct spectrophotometry of total bile acids in serum. Clin Chem 1981; 27: 1352-1356.

10 Miki K, Ichinose M, Kawamura N, et al. The significance of low serum pepsinogen levels to detect stomach cancer associated with extensive chronic gastritis in Japanese subjects. Jpn J Cancer Res 1989; 80: 111-114.

11 Matsuhisa T, Yamada N, Kato S, Matsukura N. Helicobacter pylori infection, mucosal atrophy and intestinal metaplasia in Asian populations: a comparative study in age-, gender- and endoscopic diagnosis-matched subjects. Helicobacter 2003; 8: 29-35.

12 Matsuhisa T, Matsukura N, Yamada N. Topography of chronic active gastritis in Helicobacter pylori positive Asian populations: age-, gender- and endoscopic diagnosis-matched study. J Gastroenterol 2004; 39: 324-328.

13 Woolf B. On estimating the relationship between blood group and disease. Ann Hum Genet 1955; 19: 251-253.

14 Satoh K, Kimura K, Yoshida Y, Kasano T, Kihira K, Taniguchi Y. A topographical relationship between Helicobacter pylori and gastritis: quantitative assessment of Helicobacter pylori in the gastric mucosa. Am J Gastroenterol 1991; 86: 285-291.

15 Kimura K, Satoh K, Yoshida Y, Taniguchi Y, Ido K, Takemoto T. Chronological extension of atrophic gastritis and intestinal metaplasia in normal Japanese. Eur J Gastroenterol Hepatol 1993; 5 (Suppl 1): S85-S91.

16 Matsuhisa T, Iso N, Oshima H. Endoscopic study of the form and the function of the pyloric ring. (Japanese with English abstract) Prog Dig Endosc 1987; 30: 132-136.

17 Rydning A, Berstad A. Intragastric bile acid concentrations in healthy subjects and in patients with gastric and duodenal ulcer and the influence of fiber-enriched wheat bran in patients with gastric ulcer. Scand J Gastroenterol 1985; 20: 801-804.

18 Gotthard R, Bodemar G, Tjädermo M, Tobiasson P, Walan A. High gastric bile acid concentration in prepyloric ulcer patients. Scand J Gastroenterol 1985; 20: 439-446.

\section{Abbreviations}

CI confidence interval

CLEIA chemiluminescent enzyme immunoassay

DGR duodenogastric reflux

H. pylori Helicobacter pylori

MALT mucosa-associated lymphoid tissue

OR odds ratio

PG pepsinogen

SE standard error
19 Schindlbeck NE, Heinrich C, Stellaard F, Paumgartner G, Müller-Lissner SA. Healthy controls have as much bile reflux as gastric ulcer patients. Gut 1987; 28: 1577-1583.

20 Fracchia M, Pellegrino S, Secreto P, et al. Biliary bile acid composition in gastric cancer. Int J Clin Lab Res 1999; 29: 46-48.

21 Han SW, Evans DG, el-Zaatari FAK, Go MF, Graham DY. The interaction of $\mathrm{pH}$, bile, and Helicobacter pylori may explain duodenal ulcer. Am J Gastroenterol 1996; 91: 1135-1137.

22 Offerhaus GJ, Rieu PN, Jansen JB, Joosten HJ, Lamers CB. Prospective comparative study of the influence of postoperative bile reflux on gastric mucosal histology and Campylobacter pylori infection. Gut 1989; 30: 1552 1557.

23 Chen SL, Mo JZ, Cao ZJ, Chen XY, Xiao SD. Effects of bile reflux on gastric mucosal lesions in patients with dyspepsia or gastritis. World $J$ Gastroenterol 2005; 11: 2834-2837.

24 Sakaki N, Kozawa H, Egawa N, Tu Y, Sanaka M. Ten-year prospective follow-up study on the relationship between Helicobacter pylori infection and progression of atrophic gastritis, particularly assessed by endoscopic findings. Aliment Pharmacol Ther 2002; 16 (Suppl 2): S198-S203.

25 Miki K, Ichinose M, Shimizu A, et al. Serum pepsinogens as a screening test of extensive chronic gastritis. Gastroenterol Jpn 1987; 22: 133-141.

26 Kimura K. Chronological transition of the fundic-pyloric border determined by stepwise biopsy of the lesser and greater curvatures of the stomach. Gastroenterology 1972; 63: 584-592.

27 Satoh K, Kimura K, Yoshida Y, et al. Relationship between Helicobacter pylori and atrophic gastritis. Eur J Gastroenterol Hepatol 1994; 6 (Suppl 1): S85-S88.

28 Nakamura K, Takizawa T. Pathological aspects of chronic gastritis. Definition and relationship with cancer. Gastrointestinal function, In: Kasuya Y, Tsuchiya M, Nagao F and Matsuo Y, eds. Regulation and disturbances vol. 14, Excepta Media, Tokyo, 1986; 52-63.

29 Matsukura N, Suzuki K, Kawachi T, et al. Distribution of marker enzymes and mucin in intestinal metaplasia in human stomach and relation to complete and incomplete types of intestinal metaplasia to minute gastric carcinomas. $J$ Natl Cancer Inst 1980; 65: 231-240.

30 Uemura N, Okamoto S, Yamamoto S, et al. Helicobacter pylori infection and the development of gastric cancer. N Engl J Med 2001; 345: 784-789.

31 Antonioli DA. Precursors of gastric carcinoma: a critical review with a brief description of early (curable) gastric cancer. Hum Pathol 1994; 25: 994-1005.

32 Tatemastu M, Furihata C, Katsuyama $\mathrm{T}$, et al. Independent induction of inteatinal mataplasia and gastric cancer in rats treated with $N$-methyl- $N$ '-nitro$N$-nitrosoguanitidine. Cancer Res 1983; 43: 1335-1341.

33 Tsutsumi Y. Pathophysiological significance of intestinal metaplasia of the gastric mucosa. An immunohistochemical approach. Gastrointestinal function. In: Kasuya Y, Tsuchiya M, Nagao F and Matsuo Y, eds. Regulation and disturbances vol. 14, Excepta Medica, Tokyo, 1986; 15-37.

$34 \mathrm{Xu} \mathrm{Y,} \mathrm{Watanabe} \mathrm{T,} \mathrm{Tanigawa} \mathrm{T,} \mathrm{et} \mathrm{al.} \mathrm{Bile} \mathrm{acids} \mathrm{induce} \mathrm{Cdx2} \mathrm{expression}$ through the farnesoid $\mathrm{X}$ receptor in gastric epithelial cells. $J$ Clin Biochem Nutr 2010; 46: 81-86.

35 Mason RC. Duodenogastric reflux in rat gastric carcinoma. Br J Surg 1986; 73: $801-803$. 